\title{
Efficacy of subcutaneous Immunotherapy in Allergic Rhinitis: The Grampian experience
}

\section{A Gomati'1, R Dwivedi1', V Vallamkondu, N Youakim, B Ram, S Maini \\ Department of Otolaryngology and Head Neck Surgery, Aberdeen Royal Infirmary, UK}

\section{Introduction:}

Allergic rhinitis has a detrimental effect on the quality of life and productivity of affected individuals. It's $s$ a significant public health concern. AR "Allergic Rhinitis" affects more than 400 million people worldwide; in Europe, prevalence ranges between $17 \%$ and $29 \%$. 1,2

Based on the ARIA criteria, AR was moderate to severe in $80.3 \%$ of cases, and persistent in $65.8 \%$ despite maximal pharmacotherapy. ${ }^{1}$

The standard therapy for AR includes allergen avoidance, intranasal corticosteroids and intranasal / systemic antihistamines. In spite of a plethora of pharmacological agents available, a third of children and almost two thirds of adults with AR report only partial or poor relief with pharmacotherapy.

Allergen specific / Immunotherapy (IT) is the only diseasemodifying treatment for allergic rhinitis. The first clinical description of IT was made by Noon and Freeman in 1911 in grass pollen allergic rhinitis. Allergen immunotherapy is a form of treatment that involves administering repetitive doses of allergen over time to induce immunological tolerance - the ability to inhale the antigen without allergic symptoms after discontinuation of the therapy. The two common methods of allergen administration are the subcutaneous route (subcutaneous immunotherapy, SCIT) and the sublingual route (sublingual immunotherapy, SLIT). ${ }^{3,4}$

\section{Aims:}

To evaluate efficacy of subcutaneous immunotherapy in our cohort of patients at Aberdeen Royal Infirmary.

\section{Methods:}

A cross-sectional study, carried out through face-to-face interviews, with outcomes being symptom severity recorded using a $10 \mathrm{~cm}$ visual analogue scale and percentage in reduction of medication usage.

\section{Demographics:}

Total cohort in the department where 33 patients (27 had at least 1 cycle complete with responses, 6 had $1^{\text {st }}$ cycle, response awaited, 2 pregnant and 4 uncontactable).

Therefore total patients included are 21 patients, eleven of which suffer from polyallergies; with a male to female ratio of $1: 1.1$ and a mean age of 36.5 years (SD - 11 years).

Two of our patients had completed three cycles of Immunotherapy, eleven completed two cycles and eight completed a single cycle.

\section{References:}

[1] Brozek JL, Bousquet J, Baena-Cagnani CE, et al. Allergic Rhinitis and its Impact on Asthma (ARIA) guidelines: 2010 evision. J Allergy Clin Immunol 2010;126:466-76.

2] Bauchau V, Durham SR. Prevalence and rate of diagnosis of allergic rhinitis in Europe. Eur Respir J 2004:24:758-64 [3] Freeman, J. Further observations of the treatment of hay fever by hypodermic inoculations of pollen vaccine. Lancet [4] Noon, L. Prophylactic inoculation against hay fever. Lancet 1911;177:1572-1573.

\section{Results:}

The main four domains recorded for symptoms where: nasal, eye, eczema and asthma symptoms. With 3 outcomes measured for reduction in usage of pharmacotherapy including oral anti-histamines, nasal sprays and asthma inhalers.

This showed remarkably promising results :-

Eye symptoms $81.0 \%(n=17)$

Asthma 47.6\% $(n=10)$

Eczema $33.3 \%(n=7)$

$\%$ reduction in antihistamines $71.4 \%(n=15)$, (by 35\%)

$\%$ reduction in nasal spray use $66.0 \%(n=14)$, (by $35 \%)$

$\%$ reduction in asthma inhalers $19.0 \%(4)$, (by $20 \%$ )

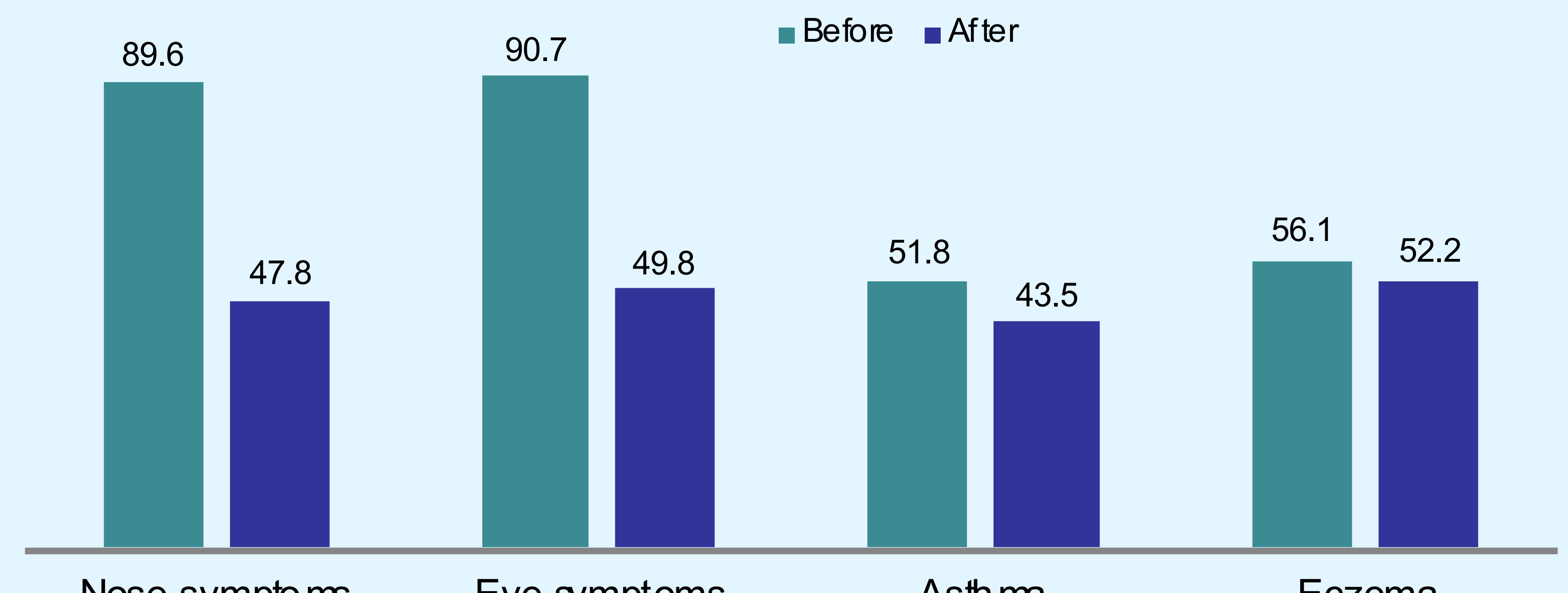

Nose sympto ms

Eye symptoms

Asth ma

Eczema

Symptom scores (total cohort)

Polyallergic - Monoallergic

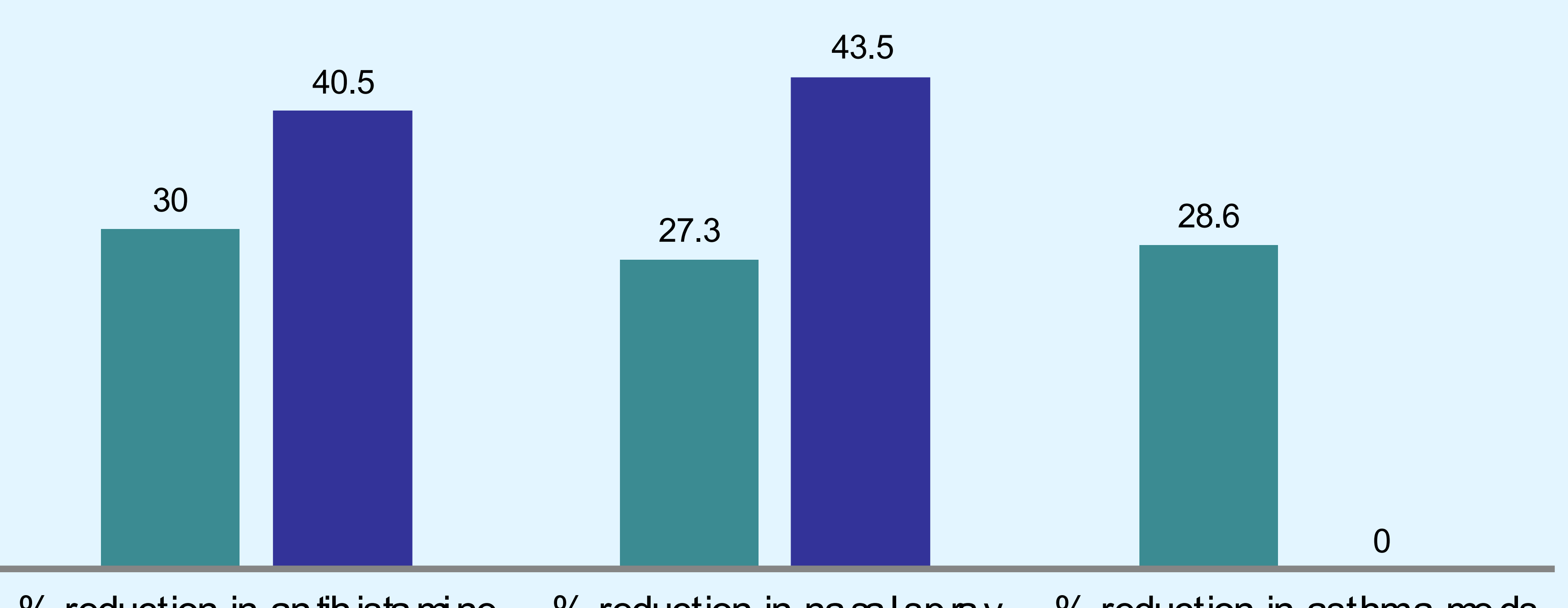

$\%$ reduction in medication usage

(Poly vs. Mono allergic)

\section{Conclusion:}

This study provides a unique perspective on outcome measures and the role of immunotherapy in managing allergic rhinitis. These results have shown effectivity of this disease-modifying treatment in our cohort.

The field of immunotherapy for $A R$ is wide open for further research and improvement in treatment modalities and one of the key research areas would include further understanding of the immunological mechanisms that induce tolerance to allergens following immunotherapy and the development of biomarkers to measure the patient's response to treatment. 